\title{
Genexpert rapid detection of rifampicin resistance among patients with pulmonary tuberculosis
}

\author{
GILDA GEORGETA POPESCU ${ }^{1,2}$, ADRIANA MOISOIU ${ }^{1}$, ARIADNA \\ PETRONELA FILDAN ${ }^{3 *}$, DOINA ECATERINA TOFOLEAN ${ }^{3}$, IOAN \\ ANTON ARGHIR ${ }^{3}$, ILEANA ION ${ }^{3}$, OANA CRISTINA ARGHIR ${ }^{3}$ \\ ${ }^{1}$ Marius Nasta Institute of Pneumology, Bucharest, Romania, 90 Viilor Str, 050152,
Bucharest, Romania
${ }^{2}$ Titu Maiorescu University of Medicine and Pharmacy, Bucharest, 22 Dambovnicului
Tineretului Str, 040441, Bucharest, Romania
${ }^{3}$ Ovidius University of Constanta, Faculty of Medicine, 124 Mamaia Blvd, 900527,
Constanta, Romania
}

Abstract

The routine conventional phenotypic bacteriological tests available for pulmonary tuberculosis (PTB) diagnosis, such as microscopy by Ziehl Neelsen staining, and Lowenstein Jensen method of solid culture, requires a long time for revealing positivity, resulting in a delayed diagnosis. After Mycobacterium tuberculosis (MTB) genome was discovered, nucleic acid amplification techniques were developed with more rapid detection and identification of rifampicin resistance in respiratory and extra-pulmonary specimens. GeneXpert is a DNA-polymerase chain reaction technique based on NAA, which allows an accurate genotypic method for a quick diagnosis of PTB diagnosis. The aim of the study was to assess retrospectively the yield of genotypic assay GeneXpert in revealing multidrug resistant TB compare to phenotypic confirmation by solid culture.

512 patients with positive and negative smears of suspected PTB were investigated by conventional phenotypic assays. PTB diagnosis assessed by positive genotypic assay and confirmed by phenotypic conventional solid culture method was $78.12 \%$. The yield of GeneXpert in revealing rifampicin resistance was high $(n=79 / 512 ; 15.43 \%)$ with $12.5 \%$ additional resistance against isoniazid revealed by positive solid cultures and drug susceptibility test. GeneXpert assay is a very useful method for rapid detection of MTB and drug resistance, which facilitates timely diagnosis and appropriate management of pulmonary tuberculosis.

Keywords GeneXpert, rifampicin, drug resistance, pulmonary tuberculosis.

To cite this article: POPESCU GG, MOISOIU A, FILDAN AP, TOFOLEAN DE, ARGHIR IA, ION T, ARGHIR OC. Genexpert rapid detection of rifampicin resistance among patients with pulmonary tuberculosis. Rom Biotechnol Lett. 2021; 26(1): 2283-2288. DOI: 10.25083/rb1/26.1/2283.2288 


\section{Introduction}

According to 2018 Global TB Report of World Health Organization, Pulmonary (P) Tuberculosis (TB) is a very frequent contagious chronic disease, diagnosed in almost 10 million humans, and one of the leading causes of death in the whole world, killing 1.6 million of people in 2017 [1]. Only a relatively small percentage (5-10\%) of the estimated 1.7 billion individuals infected with Mycobacterium tuberculosis (MTB) will progress to TB disease during their lifetime [2], but the risk of TB infection progression is much higher in immunosuppressed persons [3]. Tuberculosis is one of the major infectious diseases with predominant involvement of lung. Since Robert Koch discovered, in 24 March 1882, the agent of TB disease, Koch bacillus, several phenotypic and genotypic techniques of TB diagnosis have been developed to rapid detection of mycobacteria in sputum and extra-pulmonary specimens. As an alternative of phenotypic methods, genotypic molecular assays demonstrated their specificity, efficacy and capacity for rapid diagnosis of pulmonary TB (PTB) and drug resistant (DR) TB. There are several routine conventional phenotypic bacteriological tests available for PTB diagnosis like microscopy by Ziehl Neelsen (ZN) staining, with relative low sensitivity, and Lowenstein Jensen method of solid culture, which requires a long time for revealing positivity.

After MTB genome was discovered, nucleic acid amplification (NAA) techniques were developed with more rapid detection and identification of rifampicin resistance (RR) in respiratory and extra-pulmonary specimens [4]. GeneXpert (Xpert ${ }^{\circledR}$ MTB/Rif assay), a DNA-PCR technique based on NAA, having an equipment completly automated, closed, compact system, and endorsed by World Health Organization (WHO) as an accurate genotypic method for TB diagnosis [5, 6]. Its accuracy for early diagnosis and rapid detection of resistant strains of Mycobacterium tuberculosis (MTB) is useful in a country with a high burden of TB disease [7-10].

The aim of the study was to assess retrospectively the yield of genotypic assay GeneXpert in revealing multidrug resistant (MDR) TB compare to phenotypic confirmation by solid culture on Lowenstein Jensen.

\section{Materials and Methods}

Considering the recommendations of the Romanian National Strategy of Preventing, Surveillancing and Controling TB, one year retrospective study was performed, in 2018, in the Reference Tuberculosis Laboratory of «Marius Nasta» Pulmonology Institute, Bucharest, Romania. Study population was represented by all TB suspected patients with referrals to $4^{\text {th }}$ Sector TB Unit of Bucharest, Romania, considering their respiratory and/or systemic symptoms and signs of TB disease as prolonged cough, with or without expectoration for more than 3 weeks, fatigue, hemoptysis, weight loss, loss of appetite, as well as abnormal chest films. All eligible subjects signed the informed consent of the procedures and accepted their data to be used for scientific use, according to the revised 2000 World Medical Association Declaration of Helsinki. Related information about age, gender and bacteriological exam were assessed. Every two specimens of sputum collected from each TB suspected patient were firstly investigated by conventional method of Ziehl Neelsen (ZN) staining and direct smear microscopy screening for acid fast bacilli (AFB). This rapid method of detecting AFB can reveal positive slides with AFB presenting as red colored bacilli or negative slides with no AFB.

A total of 2,246 specimens were later reviewed for the detection of Mycobacterium tuberculosis Complex and Rifampicin Resistance (RR) by GeneXpert, a molecular rapid assay. The GeneXpert equipment is a completly automated, closed, compact system. The pathological product, the sputum, is introduced in the plastic cardridge of the equipment. The duration of the sample processing is approximatively 90 minutes. The result is displayed on the laptop connected to the equipment. Genotyping performance in revealing MTB, in 512 patients, was compared to the yield of classic phenotypic method of Lowenstein Jensen solid cultures. Results were categorized in positive smears and cultures $(\mathrm{M}+\mathrm{C}+)$, negative smears and positive cultures $(\mathrm{M}-\mathrm{C}+)$, negative smears and cultures (M-C-) and possible false positive smears (M+ C-). Drug susceptibility test (DST) was done for all positive solid culture. All procedures performed in this study were in accordance with ethical standards.

Statistical analysis was performed by version 17 of Software Package Statistical Analysis (SPSS), applying table of contingency analysis, considering OR as odd ratio and RR as relative risk, and evaluating significant results if $\mathrm{p}<0.005$. The yield of molecular GeneXpert method (high, moderate, low and very low) in detecting Pulmonary Tuberculosis active disease was assessed by the true or false positive samples revealed by the conventional phenotypic bacteriological examination.

\section{Results}

Mycobacterium tuberculosis complex (MTBC) was identified by GeneExpert molecular method in 354 males and 158 females, with a 2.24 gender ratio males/females. MTB molecular detection was assessed both in children and elders, limits from 1 to 97 years, with a significant different distribution by age and gender, between men and women $\left(\mathrm{chi}^{2}=46.6890 ; \mathrm{p}<0.0001\right)$, with a higher incidence in young women compare to young men. GeneXpert testing performed, from January to December 2018 , in Ambulatory TB Unit of $4^{\text {th }}$ Sector of Bucharest, showed high $(\mathrm{n}=190 / 512 ; 35.93 \%)$, medium $(\mathrm{n}=88 / 512$; $17.18 \%)$, low $(n=129 / 512 ; 25.39 \%)$, very low $(n=60 / 512$; $12.69 \%$ ), and visible traces of MTBC molecular detection in other 45 patients (Table 1). In the group with high MTBC detection $(n=190), 97.28 \%$ patients had both positive AFB smears and solid cultures $(n=179)$ compare to $41.53 \%$ in the very low detection group $(n=27)$. A few cases $(n=112$; $21.88 \%$ ) had neither positive AFB smear nor mycobacterial positive cultures. So, the percentage of negative 
phenotypic diagnosis of TB increased up to $58.47 \%$ in cases with very low MTBC molecular detection $(n=33)$ (Table 1). The distribution of both positive conventional phenotypic investigation by Ziehl Nielsen staining, direct smear microscopic screening for AFB, and solid culture method was noticed in most of the cases $(n=400 ; 78.12 \%)$ but the yielding of high level detection by GeneXpert is significantly influenced by the positive MICROSCOPIC exam $(169 / 179$ versus $17 / 27$; OR=9.9412; $R R=1.4995$; $\left.\mathrm{chi}^{2}=23.0063 ; \mathrm{p}=0.0000261\right)($ Table 1$)$.

Table 1. Levels of Mycobacterium tuberculosis complex (MTBC) detection in sputum by genotypic GeneXpert compare to conventional phenotypic assay (microscopic exam of acid bacilli fast smears and mycobacterial solid cultures)

\begin{tabular}{|c|c|c|c|c|c|}
\hline Genotypic & \multicolumn{4}{|c|}{ Phenotypic Assay } & \multirow[t]{3}{*}{ Cases } \\
\hline Positive & \multicolumn{2}{|c|}{ Positive } & \multicolumn{2}{|c|}{ Negative } & \\
\hline $\begin{array}{l}\text { Levels of } \\
\text { MTBC } \\
\text { detection }\end{array}$ & $\begin{array}{l}\text { Positive smears and } \\
\text { cultures }(M+C+)\end{array}$ & $\begin{array}{c}\text { Negative smears and } \\
\text { positive cultures (M- } \mathrm{C}+\text { ) }\end{array}$ & $\begin{array}{l}\text { Negative smears and } \\
\text { cultures (M- C-) }\end{array}$ & $\begin{array}{c}\text { False positive } \\
\text { smears (M+C-) }\end{array}$ & \\
\hline \multirow{2}{*}{$\begin{array}{c}\text { High } \\
(\mathrm{N} ; \%)\end{array}$} & 169 & 10 & 10 & 1 & 190 \\
\hline & \multicolumn{2}{|c|}{$179 ; 97.28$} & \multicolumn{2}{|c|}{$11 ; 2.72$} & \\
\hline \multirow{2}{*}{$\begin{array}{c}\text { Medium } \\
(\mathrm{N} ; \%)\end{array}$} & 68 & 14 & 6 & 0 & 88 \\
\hline & \multicolumn{2}{|c|}{$82 ; 93.18$} & \multicolumn{2}{|c|}{$6 ; 6.82$} & \\
\hline \multirow{2}{*}{$\begin{array}{c}\text { Low } \\
(\mathrm{N} ; \%)\end{array}$} & 57 & 37 & 32 & 3 & 129 \\
\hline & \multicolumn{2}{|c|}{$94 ; 72.30$} & \multicolumn{2}{|c|}{$35 ; 27.70$} & \\
\hline \multirow{2}{*}{$\begin{array}{l}\text { Very low } \\
(\mathrm{N} ; \%)\end{array}$} & 17 & 10 & 23 & 10 & 60 \\
\hline & \multicolumn{2}{|c|}{$27 ; 41.53$} & \multicolumn{2}{|c|}{$33 ; 58.47$} & \\
\hline \multirow{2}{*}{$\begin{array}{l}\text { Traces } \\
(\mathrm{N} ; \%)\end{array}$} & 4 & 14 & 25 & 2 & 45 \\
\hline & \multicolumn{2}{|c|}{$18 ; 40$} & \multicolumn{2}{|c|}{$27 ; 60$} & \\
\hline \multirow{2}{*}{$\begin{array}{l}\text { Total cases } \\
(\mathrm{N} ; \%)\end{array}$} & 315 & 85 & 96 & 16 & 512 \\
\hline & \multicolumn{2}{|c|}{$400 ; 78.12$} & \multicolumn{2}{|c|}{$112 ; 21.88$} & \\
\hline
\end{tabular}

There is a significant difference in MTB detection level provided by GeneXpert in positive AFB smears compare to negative ones, according to phenotypic investigation of sputum by $\mathrm{ZN}$ staining and solid Lowenstein Jensen cultures $\left(\mathrm{chi}^{2}=5540.74 ; \mathrm{p}<0.0001\right)$ (Figure 1$)$, with a significant higher levels of MTB molecular detection in adults over 50 and below 60 years old $\left(\mathrm{chi}^{2}=56.4850\right.$; $\mathrm{p}=0.0001)$ (Figure 2).

GeneXpert assay detected $15.43 \% \quad(n=79 / 512)$ resistance against Rifampicin (RR). Only $12.5 \%(n=64)$ of RR cases were identified with supplementary resistance against isoniazid (MDR pattern) by DST performed by positive solid culturing. Phenotypic DST was done in all patients with positive cultures and $10.35 \%(n=53 / 512)$ were resistant to both Rifampicin and Isoniazid.

\section{Discussions}

According to the European Center of Disease Prevention and Control (ECDC) 2018 Report, in Romania, there were diagnosed 13,617 TB cases, in $2016,88.7 \%$ were drug sensitivity tested and the notified MDR-TB cases represented 5.6\% of cases (458) [8]. Out of 458 Romanian MDR-TB cases, only 199 (37.4\%) were successfully treated, 156 failed (29.3\%), and 93 died (17.5\%), so MTR-TB can contribute to elevated TB mortality rate [10].

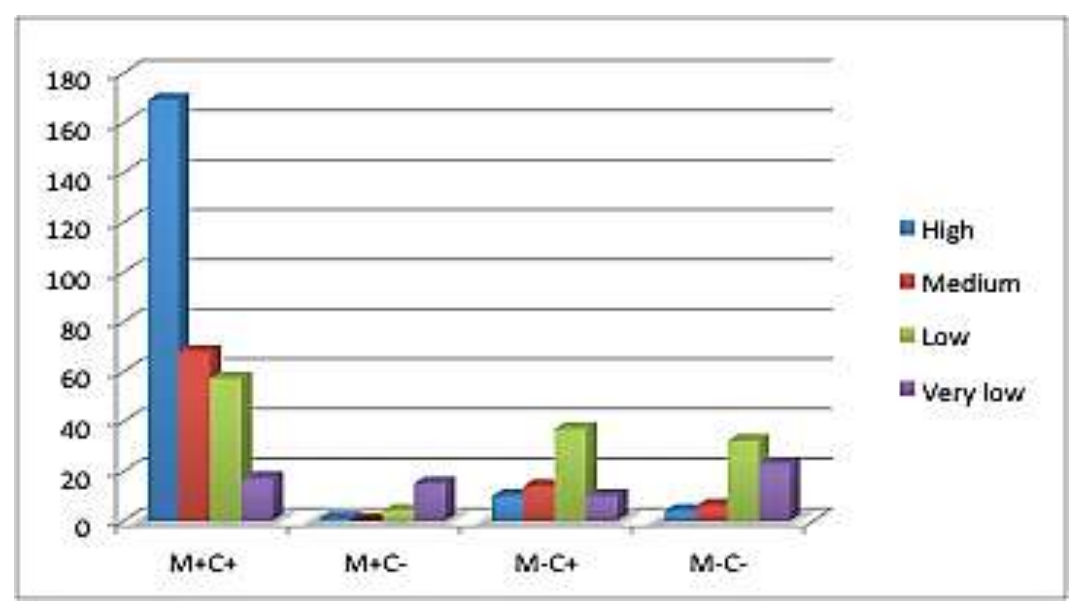

Figure 1. The distribution of positive GeneXpert results by different levels of Mycobacterium tuberculosis detection according to conventional microscopic (M) and culture (C) phenotypic methods. 


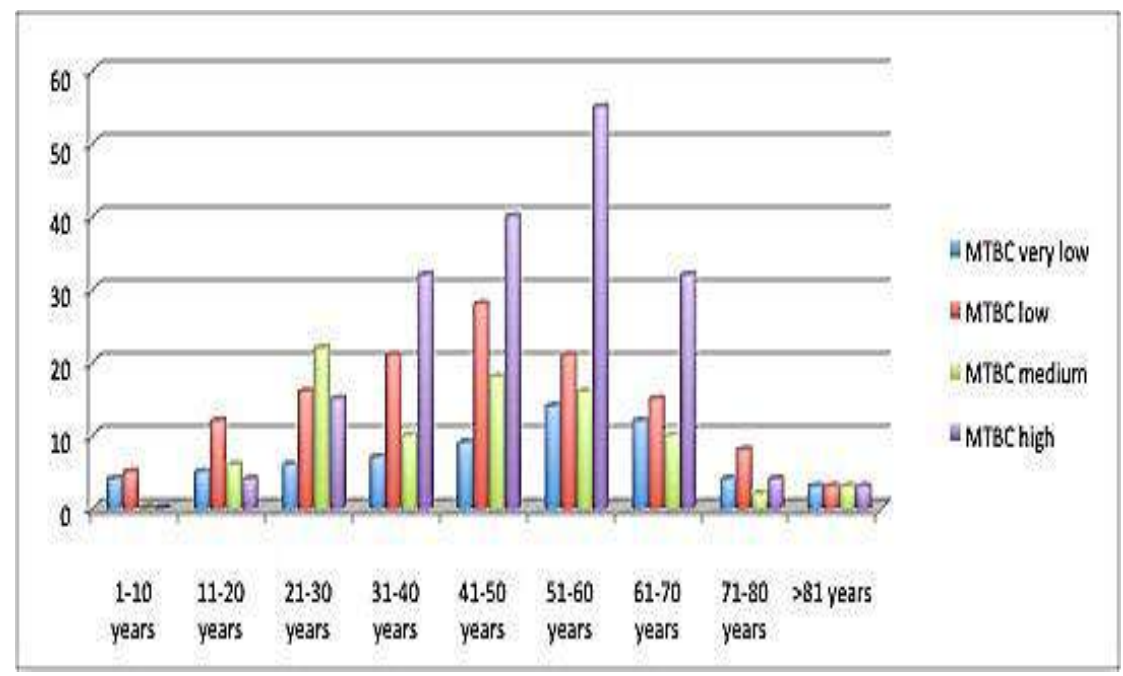

Figure 2. The distribution of GeneXpert levels of Mycobacterium tuberculosis complex (MTBC) detection by the age of cases.

According to the End TB Strategy, the WHO approved and recommended all positive microscopic examinations to be molecular investigated for rapid detecting of MTB resistance against to Rifampicin (RMP) and, therefore, potential TB MDR must be considered because RMP resistance is a MDR predictor [11]. Mycobacterium tuberculosis can develop multidrug resistant (MDR) strains to isoniazid $(\mathrm{H})$ and rifampicin $(\mathrm{R})$ with or without resistance to other drugs. Rifampicin-resistant TB (RR-TB) is defined as resistance only to rifampicin, with or without resistance to other first-line anti-TB drugs [1]. MDR-TB and RR-TB represent a huge human health threat to infection and disease prevention, control and surveillance. MDR-TB is still a treatable disease but therapy includes toxic drugs, which are also very expensive, requiring a very long time of administering. The Global TB Report 2018 estimated that MDR-TB represented $82 \%$ of RR-TB cases $(558,000)$, diagnosed in 2017 [1]. In low incidence countries, migration has a significant impact on MDR-TB [10]. In Romania, MTB phenotypic testing for the sensitivity to anti-tuberculosis drugs, as isoniazid ( $\mathrm{INH}, \mathrm{H}$ ) and rifampicin (RMP, R), is performed by the absolute concentration method and the rapid phenotypic assay of Nitrate Reductase. In the reference Romanian laboratories, the method of Löwenstein Jensen (LJ) solid culture is used to drug sensitivity phenotypic testing. Revealing the resistance of MTB against first and second antiTB drugs line by BACTEC MGIT 960, an automated liquid medium of culture for MTB, is implemented in all counties of Romania. Phenotypic DST for INH and RMP is a routine method performed in laboratories, which all have implemented the quality management system constituting a safety feature, but results could be false negative or false positive by the intervention of several factors, as incorrect inoculation of sputum, lack of representativeness and age of culture, stability of anti-tuberculosis drugs in the medium of culture, use of inadequate media or laboratory kits, lack of experience in performing and/or interpreting the results.

The available molecular tests in Romania are more applicable to positive than to negative products on microscopic examination. Two commercial linear hybridization tests are used for early MTB detection in various biological specimens such as sputum, bronchial aspiration, tissue, pleural fluid. GeneXpert MTB / RIF, a closed, fully automated system for simultaneous low to high detection and identification of Mycobacterium tuberculosis complex and RMP resistance (RR) by a Real Time PCR technology [12], use single-use cartridges in a product-specific platform, which allows the analysis of amplified genetic fragments and offers results in less than 2 hours. Line Probe Assay (LPA), based on a Polymerase Chain Reaction (PCR), is used to amplify certain regions of the mycobacterial genome, continuing with reverse-hybridization to identify sample-specific sequences [13] is capable to simultaneously detection of MTB complex and MTB resistance against HR. GenoType MTBDRplus test can detect common mutations in the rpoB gene (responsible for RMP resistance) and in the katG genes (responsible for INH resistance) [13] after a mean test time of two days when applied directly to the sputum. The genotypic assay does not eliminate the need for conventional phenotypic culture and DST, which are necessary for second line antiTB drugs testing.

For preventing delayed diagnosing of TB and interpreting the results of genotypic methods, both the laboratory specialist and the clinician should be aware of the yield of every assay compare to phenotypic investigation [14]. In our study, male predominance is confirming the epidemiological data of higher rates of PTB in males compare to females $[1,9]$. The high rate of TB occurrence in older people sustains the previous WHO estimations, in 2002, of future geriatric TB disease profile [15], and of numerous other age- related pulmonary diseases. There 
are cases of similarities with other lung granulomatosis diseases [16], or masquerading comorbidities [17], or unidentified PTB revealed postmortem [18], so, molecular markers of PTB as GeneXpert can be useful in diagnosing the etiology of TB [19]. Analyzing the concordance between the genotypic and phenotypic methods, it seems that rapid molecular detection has a high rate of phenotypic confirmation. Similar results of were found in different studies [20, 21], clearly showed GeneXpert has high sensitivity and specificity for detecting MTB complex in pulmonary samples of patients with PTB. Progress in molecular biology and rapid phenotypic assays has provided new possibilities for detecting and identifying micro-organisms, especially in a TB burden country, like Romania, helping pulmonologist in differential diagnosis of TB with lung cancer or other chest tumors, or rare diseases mimicking TB. The development of molecular cloning techniques has allowed the characterization of DNA fragments, which can be used in hybridization tests to detect specific organisms, and methods of rapid DNA sequencing have facilitated the creation of specific probes. In addition, molecular approaches revolutionized epidemiology of TB. Currently, highly specific markers for the subtyping of Mycobacterium tuberculosis strains are, also, available to clarify recent problems such as TB spread in HIV-positive individuals including the selection and spread of multi-resistant strains of MTB [22]. Persistence of respiratory symptoms or deteriorating of the health status and/or the decline of lung function, as documented for TB [23], can have medium and long term impact on the quality of life. Therefore, the most adequate treatment based on genetic test results is essential to achieve a better quality of life of the TB patients.

\section{Conclusions}

TB diagnosis, assessed by positive genotypic assay and confirmed by phenotypic conventional method, was $78.12 \%$. The yield of GeneXpert in revealing the Rifampicin Resistance among patients with Pulmonary Tuberculosis was high $(15.43 \%)$, with an additional resistance against isoniazid (12.5\%) revealed by positive solid cultures and DST. GeneXpert assay is a very useful method for rapid detection of MTB and drug resistance, which facilitates timely diagnosis and appropriate management of pulmonary tuberculosis.

\section{Conflicts of Interest}

None to declare

\section{Abbreviations}

$\mathrm{AFB}=$ acid fast bacilli, $\mathrm{C}=$ culture, $\mathrm{DR}=\mathrm{drug}$ resistant, DST=drug susceptibility test, INH=Isoniazid, $\mathrm{M}=$ microscopy, $\mathrm{MDR}=$ multidrug resistant, $\mathrm{MTB}=\mathrm{Myco}-$ bacterium tuberculosis, NAA=nucleic acid amplification, $\mathrm{PCR}=$ polymerase chain reaction, $\mathrm{PTB}=$ pulmonary tuberculosis, RMP=Rifampicin, RR=Rifampicin-resistant, $\mathrm{TB}=$ tuberculosis, $\mathrm{ZN}=$ Ziehl Neelsen.

\section{References}

1. WORLD HEALTH ORGANIZATION. Global tuberculosis report 2019 (WHO/HTM/TB/2019.22). Geneva: World Health Organization; 2019.

2. SLOOT R, SCHIM VAN DER LOEFF MF, KOUW PM, BORGDORFF MW. Risk of tuberculosis after recent exposure. A 10-year follow-up study of contacts in Amsterdam. Am J Respir Crit Care Med. 2014; 190: 1044-52.

3. NARASIMHAN P, WOOD J, MACINTYRE CR, MATHAI D. Risk factors for tuberculosis. Pulm. Med. 2013: 828939.

4. PIRSIMONI C, SCARPARO C, PICCOLI P, et al. Performance assessment of two commercial amplification assays for direct detection of Mycobacterium tuberculosis complex from respiratory and extrapulmonary specimens. J Clin Microbiol. 2002; 40: 4138-42.

5. BOWLES EC, FREYEE B, VAN INGEN J, MULDER $\mathrm{B}$, et al. Xpert MTB/RIF ${ }^{\circledR}$, a novel automated polymerase chain reaction-based tool for the diagnosis of tuberculosis. Int J Tuberc Lung Dis. 2011; 15: 988-9.

6. WORLD HEALTH ORGANIZATION (WHO): Automated real-time nucleic acid amplification technology for rapid and simultaneous detection of tuberculosis and rifampicin resistance: Xpert mtb/rif system. Policy statement 2011.

7. CIORAN N, POPESCU G, MIHAILESCU L, DIDILESCU C et al. TB among prisoners in Romania, 2009-2011. Eur Resp J. 2013; 42(57): P4472.

8. EUROPEAN CENTER OF DISEASE PREVENTION AND CONTROL (ECDC) 2018 Report; Tuberculosis surveillance and monitoring in Europe 2018, 2016 Romania data.

9. ARGHIR OC, DANTES E, OTELEA M, RASCU A et al. Eight year survey of tuberculosis in-hospital mortality in the South Eastern part of Romania. Rom J Leg Med. 2018; 26(2): 183-187.

10. ARGHIR OC, ALVES PEREIRA PM, RASCU A, DANTES E et al. The Impact of Migrant Tuberculosis on the Chimioresistant Pattern of Antituberculosis Drugs in a Low Burden TB European Country. Farmacia. 2018; 66(3): 537-540.

11. GILPIN C, KOROBITSYN A, WEYER K. Current tools available for the diagnosis of drug-resistant tuberculosis. Ther Adv Infectious Dis. 2016; 3(6): 145-151.

12. NICOL MP, WHITELAW A, STEVENS W. Using Xpert MTB/RIF. Curr Respir Med Rev. 2103; 9: 187-192.

13. HILLEMANN D, RUSCH-GERDES S, RICHTER E. Evaluation of the GenoType MTBDR plus Assay 
for Rifampin and Isoniazid Susceptibility Testing of Mycobacterium tuberculosis Strains and Clinical Specimens. J Clin Microbiol. 2007; 45(8): 2635-40.

14. LEYLABADLO HE, KAFIL HS, YOUSEFI M, AGHAZADEH $\mathrm{M}$ et al. Pulmonary Tuberculosis Diagnosis: Where We Are? Tuberc Respir Dis. 2016; 79: 134-142.

15. WORLD HEALTH ORGANIZATION (WHO) Report 2002 Global Tuberculosis Control-Surveillance, Planning, Financing.

16. POP M, GHIGOLEA I, MUREŞAN A, RÂJNOVEANU R et al. Tuberculosis or sarcoidosis. Rev Med Chir Soc Med Nat Iasi. 2008; 112(4): 959-964.

17. POSTOLACHE P, IANOSI ES, JIMBOREAN G. Systemic Lupus Erythematosus Flared up after Rifampicin during Active Pulmonary Tuberculosis. Rev. Chim. 2017; 68(9): 2160-2161.

18. TURCU DV, TURCANU AM, GRIGORESCU C, PATRASCU A et al. Value of Autopsies in the Study of Diagnostic Errors in Respiratory Medicine. Rev. Chim. 2019; 70(3): 1037-1039.
19. COJOCARU V, DANTES E, NOVAC MG, IONESCU AM et al. The Value of Adenosine Deaminase Enzime Level in the Positive Diagnosis of Tuberculous Pleural Effusion. Rev. Chim. 2018; 69(12): 3688-3691.

20. AGRAWAL M, BAJAJ A, BHATIA V, DUTT S. Comparative Study of GeneXpert with ZN Stain and Culture in Samples of Suspected Pulmonary Tuberculosis. J Clin Diagn Res. 2016; 10(5): DC09-12.

21. RASOOL G, KHAN AM, MOHY-UD-DIN R, RIAZ M. Detection of Mycobacterium tuberculosis in AFB smear-negative sputum specimens through MTB culture and GeneXpert@ MTB/RIF assay. Int J Immunopathol Pharmacol. 2019; 33:2058738419827174.

22. SIA JK, RENGARAJAN J. Immunology of Mycobacterium tuberculosis Infections. Microbiol Spectr. 2019; 7(4): 10.1128/microbiolspec.GPP3-0022-2018.

23. MUÑOZ-TORRICO M, RENDON A, CENTIS R, D'AMBROSIO $\mathrm{L}$ et al. Is there a rationale for pulmonary rehabilitation following successful chemotherapy for tuberculosis? J Bras Pneumol. 2016; 42(5): 374-385. 